\title{
On-Chip Correlator for Passive Wireless SAW Multisensor Systems
}

\author{
Liqiang Xie, Tianxian Wang, Ping Wang, and Jianchun Xing \\ College of Defense Engineering, PLA University of Science and Technology, Nanjing 210007, China \\ Correspondence should be addressed to Ping Wang; wp893@sina.com
}

Received 27 March 2016; Accepted 4 July 2016

Academic Editor: Stefania Campopiano

Copyright (C) 2016 Liqiang Xie et al. This is an open access article distributed under the Creative Commons Attribution License, which permits unrestricted use, distribution, and reproduction in any medium, provided the original work is properly cited.

\begin{abstract}
For decoding the asynchronous superposition of response signals from different sensors, it is a challenge to achieve correlation in a code division multiplexing (CDM) based passive wireless surface acoustic wave (SAW) multisensor system. Therefore, an onchip correlator scheme is developed in this paper. In contrast to conventional CDM-based systems, this novel scheme enables the correlations to be operated at the SAW sensors, instead of the reader. Thus, the response signals arriving at the reader are the result of cross-correlation on the chips. It is then easy for the reader to distinguish the sensor that is matched with the interrogating signal. The operation principle, signal analysis, and simulation of the novel scheme are described in the paper. The simulation results show the response signals from the correlations of the sensors. A clear spike pulse is presented in the response signals, when a sensor code is matched with the interrogating code. Simulations verify the feasibility of the on-chip correlator concept.
\end{abstract}

\section{Introduction}

Owing to their inherent ability to work in harsh environments, completely passive wireless operation and reliable, maintenance-free life cycle, surface acoustic wave (SAW) sensors have a variety of applications, including structural health monitoring, environment sensing, and tire pressure monitoring [1-3]. Generally, these applications require several SAW sensors working together with a reader to sense specified information around. These sensors operate without any battery and communicate with the reader wirelessly. These special features can enhance the convenience and durability of many kinds of applications. A typical passive wireless SAW multisensor system is shown in Figure 1. When a signal sent from the reader reaches the sensors, it is transformed into SAW to accomplish sensing operations by each sensor. The response signals from all the sensors are fed back to the reader, for data collection. Then, a distributed monitoring task is completed by this passive wireless SAW multisensor system.

In the SAW multisensor system, a major challenge is the communication collision among sensors. The strong interference between the response signals from different sensors makes it difficult for the reader to distinguish the signals from each sensor. This collision problem is similar to that observed in wireless communication systems; and the typical methods to solve it are frequency division multiplexing, time division multiplexing (TDM), space division multiplexing, and code division multiplexing (CDM) [4]. Frequency division multiplexing, which involves allocating a unique carrier frequency to each sensor, is the method mainly used in commercial SAW sensors. However, the scarcity of frequency bands seriously restricts the capacities of multisensor systems. Based on the natural time delay of the SAW chip, TDM divides the response delay time into several slots. Each time slot is allocated to a different sensor, and the response signals from different sensors are identified by the different delays $[5,6]$. However, the delay time of a SAW sensor is restricted by the chip size and propagation loss; thus the capacity of the TDM SAW system is limited. Space division multiplexing is based on spatial focusing to minimize the number of sensors and reduce the collision probability. Therefore, the reader needs a special antenna with 15 to $18 \mathrm{~dB}$ of gain and a beam width between $20^{\circ}$ and $30^{\circ}$ to achieve spatial focusing. However, due to its principle, collisions cannot be avoided completely by this method. Code division multiplexing, used in multisensor systems, is based on the orthogonality of the sensors' codes, 


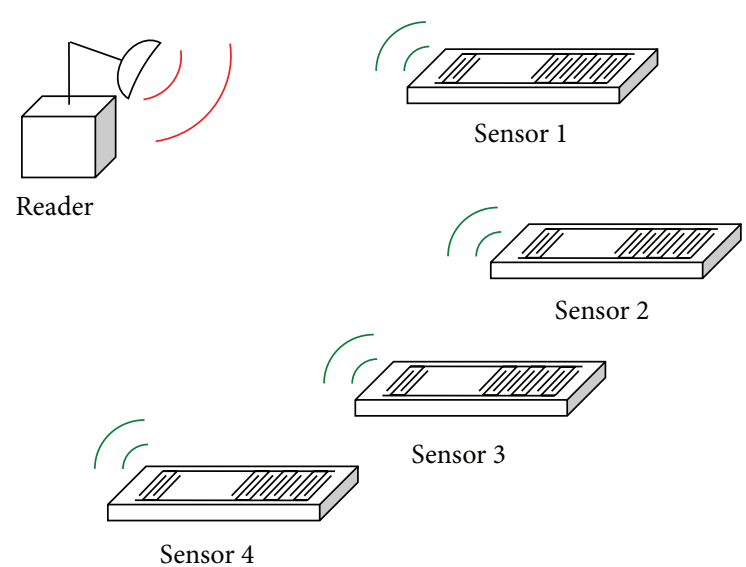

FIGURE 1: A passive wireless SAW multisensor system.

such as orthogonal frequency coding or pulse position coding, and the correlation operation takes place at the reader. To discriminate sensor codes, the reader is requested to determine the accurate arrival time of each response signal before synchronous cross-correlation detection, which is difficult for passive wireless SAW systems $[7,8]$. Therefore, the asynchronous correlation is a major challenge for the CDM-based SAW multisensor systems. Some researchers have been working on the problem of selecting a group of codes that exhibit very low cross-correlation, to accomplish asynchronous correlation operation. However, for a limited code length, it is difficult to select a large code set [9].

To solve the asynchronous correlation operation, this paper presents a correlator integrated on a SAW sensor chip, which is allocated with a unique code in an orthogonal code set. The correlation operation is achieved at each sensor instead of the reader. The reader sends an interrogating signal corresponding with a sensor code and modulated by the carrier wave. For the orthogonality of the sensors' codes, correlation is achieved undisturbedly by the correlator on each sensor chip, respectively. Then the responses of the sensors in the coverage of the interrogating signal are fed back to the reader. The sensor which corresponds with the interrogating signal is determined from the results of the correlation. Therefore, the on-chip correlator method presented in this paper is suitable for passive wireless SAW multisensor systems.

\section{Operation Principle}

The operation principle of the novel SAW multisensor system is shown in Figure 2. The reader can achieve the generation and transmission of interrogating signal, the reception of response signals from sensors, and the decision of a matched sensor. Each sensor receives the interrogating signal, which is transformed into SAW. Then the sensing and correlation are implemented by the correlator on the sensor chip and the result of the correlation is transformed back to the reader. For the passive operation feature, all the operations on the sensor are completed by the interdigital transducers (IDTs).

The initial embodiment of a SAW sensor structure is shown schematically in Figure 3. The sensor contains two

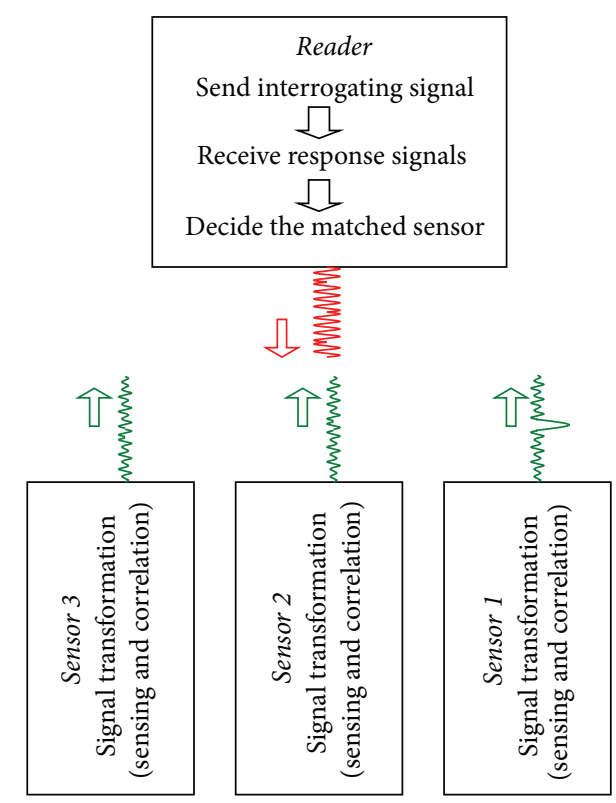

FIGURE 2: Operation principle schematic of the novel SAW multisensor system.

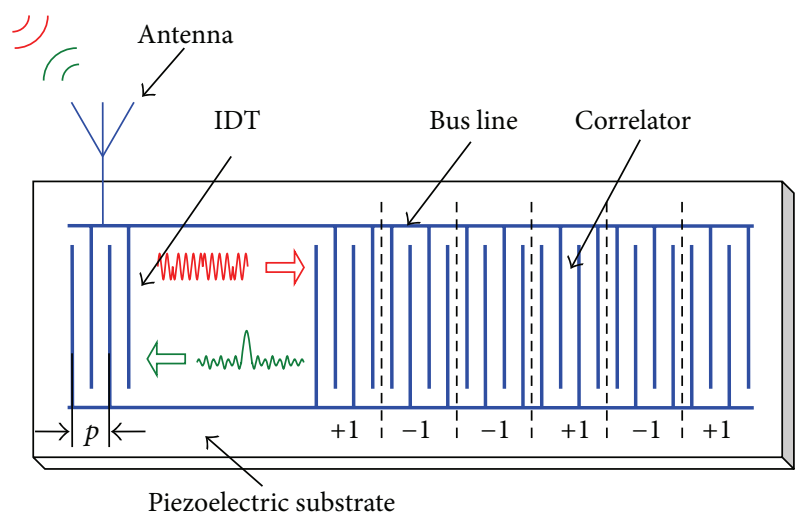

FIgURE 3: Schematic of a 6-bit code SAW sensor.

clusters of IDTs on a piezoelectric substrate. In order to reduce the losses, the structure periodicity $p$ of the IDTs is equal to the wave length $\lambda$ of the input signal with frequency $f$. All the IDTs are connected to the antenna in parallel by the bus line. In order to implement the correlation on chip, the right cluster of IDT is divided into $Z$ groups, and each group contains $h$ pairs of electrodes. $Z$ and $h$ are both integer variables. There is no gap between any adjacent groups. As shown in Figure 3, all of the sequenced IDT groups are connected to the bus line in homopolarity or heteropolarity, which is determined by a binary code allocated. For example, +1 indicates homopolarity and -1 indicates heteropolarity. In a multisenor system, the binary code of each sensor, called as sensor code, is encoded orthogonally to others. Figure 3 shows a sensor structure with code $[+1,-1,+1,-1,-1,+1]$. Therefore, the structure described above can realize correlation operation between the input code signal and the sensor code. The time-varying 
multiplication of the correlation calculation is achieved by utilizing the SAW delays and polarities of different groups of IDT. The accumulation of the multiplication results in the correlation calculation is implemented by the bus line structure connecting the IDTs.

When the system operates, the reader sends an interrogating signal with a binary code called interrogating code. The encoded interrogating signal is received by the antenna of a sensor and reaches the two clusters of IDT. Then, a series of signal transformations is done by the IDTs on the sensor chip.

On the left cluster, a SAW signal is converted from the encoded interrogating signal by the IDT and propagates to the right cluster of IDT after about a few microseconds delay time. The IDT converts the SAW signal into an electric signal, and at the same time the cross-correlation operation between the interrogating code and the sensor code is done by the structure of the right cluster of IDT. The electric signal contains the result of the cross-correlation operation.

On the right cluster, the IDT implements the crosscorrelation operation with the encoded interrogating signal firstly and launches a SAW signal corresponding to the result of the cross-correlation operation. Then the SAW signal is transduced into an electric signal by the left cluster of IDT.

The electric signals resulting from both sides of the sensor chip are summed by the bus line and resent to the reader through the antenna. For the orthogonality of the sensor codes in a multisensor system, if the interrogating code is matched with a sensor's code, then the response signal from the sensor chip shows a spike; else the response signal is expressed as noise. Therefore, the reader can discriminate the sensor which is matched with the interrogating code from the returned signal. When all codes in an orthogonal code set are sent with interrogating signal by reader separately, every sensor of the system is accessed and discriminated successfully.

Furthermore, the phase of the spike signal responded from the matched sensor can be demodulated by the reader. Then the signal sensed by the sensor is read out from the variety of the phase of the spike signal. Therefore, the interference problem among the response signals from different sensors is solved by the method of on-chip correlation operation. Then, a passive wireless SAW multisensor system is achieved by the correlator design on sensor chips.

\section{Signal Analysis}

The theory analysis presented is based on the signal transfer process of the SAW correlator. In order to execute the correlation operation, the interrogating signal is a composite of interrogating code and carrier signal. Defining the period of the carrier signal as $T$, the symbol time of the interrogating code $\tau_{c}=h \cdot T$, in accordance with the sensor structure. To simplify the analysis, the input signal of the correlator on a sensor, which is received from the interrogating signal by the antenna, can be defined as

$$
R(t)=\sum_{i=0}^{Z-1} b_{i}^{\prime} s^{\prime}\left(t-i \tau_{c}\right)
$$

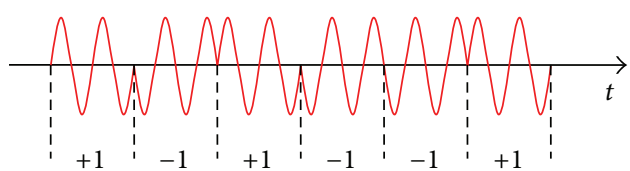

FIGURE 4: An example of the interrogating signal.

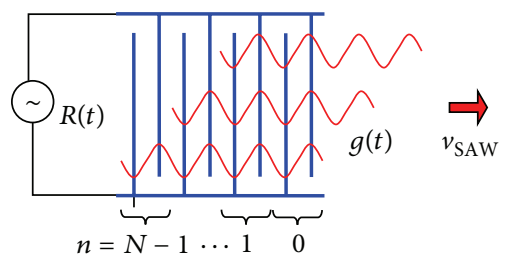

FIGURE 5: The transformation from electric signal to SAW.

$$
s^{\prime}(t)= \begin{cases}\sin (\omega t), & 0<t \leq \tau_{c} \\ 0, & \text { otherwise }\end{cases}
$$

where $s^{\prime}(t)$ is the carrier signal in the first symbol time of $R(t)$, $\left\{b_{i}^{\prime}\right\}$ is a synthesis coefficient sequence of the interrogating code and propagating loss and is designated simply as the interrogating code, and $Z$, as the number of the code symbols, is the length of the interrogating signal. When $h$ and $Z$ are each set to 2 and 6, respectively, an example of signal $R(t)$ in time domain is shown in Figure 4, and the interrogating code is set to $[+1,-1,+1,-1,-1,+1]$.

In order to deduce the analysis simply and conveniently, the input signal $R(t)$ also can be described as

$$
\begin{aligned}
& R(t)=\sum_{i=0}^{M-1} b_{i} s(t-i T), \\
& s(t)= \begin{cases}\sin (\omega t), & 0<t \leq T \\
0, & \text { otherwise }\end{cases}
\end{aligned}
$$

where $M=h \cdot Z,\left\{b_{i}\right\}$ is another expression of the interrogating code with symbol width equaling $T$ and $s(t)$ is a sine carrier signal with single period.

Based on $\delta$ function model of IDT, the periodic pairs of electrodes in an IDT are equivalent to a series of independent wave sources [10]. Then, as shown in Figure 5, the SAW signal transformed from electromagnetic wave can be simplified to be the superposition of the signals generated by all the electrode pairs of the IDT. As the frequency of the input signal $R(t)$ matches the periodicity of the IDT structure, the SAW output signal of the IDT can be deduced as

$$
g(t)=g_{0} \sum_{n=0}^{N-1} \sum_{i=0}^{M-1} b_{i} s(t-n T-i T)
$$

where $g_{0}$ is the transform coefficient of the IDT, $N$ is the number of the electrode pairs of the IDT, and $n=0$ indicates the right pair for the SAW propagating to the right. 


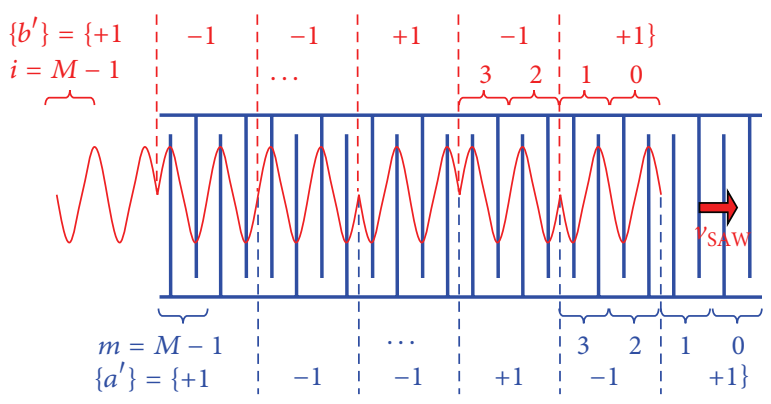

Figure 6: The transformation from SAW to electric signal.

The SAW launched by the left cluster of IDT propagates along the substrate surface to the right cluster at the velocity of $v_{\text {SAW }}$ for a distance of $d$. Assuming that the length of the sensor code is the same as that of the interrogating code $Z$, the pairs of the IDT in a symbol of the sensor code are $\tau_{c} / T=h$.

As shown in Figure 6, the right cluster of IDT detects the SAW signal and transfers it into charge signal $q(t)$ by piezoelectric effect. $q(t)$ as the summation of charges generated by all electrode pairs can be deduced as

$$
\begin{aligned}
& q(t)=\sum_{m=0}^{M-1} q_{0} a_{m} g\left(t-\frac{d}{v_{\mathrm{SAW}}}-[M-1-m] T\right) \\
& =q_{0} g_{0} \sum_{n=0}^{N-1} \sum_{m=0}^{M-1} \sum_{i=0}^{M-1} a_{m} b_{i} s\left(t-\frac{d}{v_{\mathrm{SAW}}}-[M-1-m+n+i] T\right),
\end{aligned}
$$

where $\left\{a_{m}\right\}$, as the synthesis coefficient sequence of a SAW sensor code and propagating loss, is defined simply as the sensor code with symbol width equaling $p$ and $\left\{a_{i}^{\prime}\right\}$ is another expression of the interrogating code with symbol width equaling $h \cdot p$ and $q_{0}$ is the coefficient of transform from SAW to charge signal. Setting $k=M-1-m+n+i$, then

$$
\begin{aligned}
& q(t) \\
& =q_{0} g_{0} \sum_{n=0}^{N-1} \sum_{m=0}^{M-1} \sum_{k=M-1-m+n}^{2 M-2-m+n} a_{m} b_{m+k-n-M+1} s\left(t-\frac{d}{v_{\mathrm{SAW}}}-k T\right) .
\end{aligned}
$$

For $m=0,1,2, \ldots, M-1, n=0,1,2, \ldots, N-1$, and $k$ as an integral number belongs to $[M-1-m+n, 2 M-2-m+n]$, then $k \in[0,2 M+N-3]$. Based on that fact, $b_{i}$ is set to zero, when $i>M-1$ or $i<0$. Thus,

$$
\begin{aligned}
& q(t) \\
& =q_{0} g_{0} \sum_{n=0}^{N-1} \sum_{m=0}^{M-1} \sum_{k=0}^{2 M+N-3} a_{m} b_{(m+k-n-M+1)} s\left(t-\frac{d}{v_{\mathrm{SAW}}}-k T\right) \\
& =q_{0} g_{0} \sum_{k=0}^{2 M+N-3} s\left(t-\frac{d}{v_{\mathrm{SAW}}}-k T\right) \sum_{n=0}^{N-1} \rho_{a b}(k-n-M+1),
\end{aligned}
$$

where

$$
\rho_{a b}(j)=\sum_{m=0}^{M-1} a_{m} b_{m+j}
$$

TABLE 1: Example of 16-bit sensor codes.

\begin{tabular}{lc}
\hline Sensors & Codes \\
\hline Sensor 1 & {$[+1-1+1+1-1+1-1+1+1-1-1+1-1-1-1+1]$} \\
Sensor 2 & {$[-1-1+1-1+1+1+1+1-1+1-1-1+1-1+1-1]$} \\
Sensor 3 & {$[+1+1-1-1-1+1-1-1+1-1-1+1+1-1+1+1]$} \\
Sensor 4 & {$[-1+1-1+1+1-1-1+1-1+1-1-1+1+1+1-1]$} \\
Sensor 5 & {$[+1+1+1-1-1-1+1-1+1-1+1-1-1-1+1+1]$} \\
Sensor 6 & {$[-1-1+1+1-1+1+1-1-1+1+1+1-1-1-1+1]$} \\
\hline
\end{tabular}

which is the cross-correlation function of $\left\{a_{m}\right\}$ and $\left\{b_{m}\right\}$. Then the current output of the right cluster IDT is deduced as

$$
\begin{aligned}
& i_{\text {output }}(t) \\
& \quad=q_{0} g_{0} \sum_{k=0}^{2 M+N-3} \frac{d s\left(t-d / v_{\text {SAW }}-k T\right)}{d t} \sum_{n=0}^{N-1} \rho_{a b}(k-n \\
& \quad-M+1) .
\end{aligned}
$$

Therefore, the response signal of a sensor, interrogated by the reader, is a function of cross-correlation between the interrogating code and the sensor code.

When a sensor code $\left\{a_{m}\right\}$ is identical to the interrogating code $\left\{b_{m}\right\}, \rho_{a b}(k-n-M+1)$ as the cross-correlation function of $\left\{a_{m}\right\}$ and $\left\{b_{m}\right\}$ has a clear peak at $k-n-M+1=0$. Then, $n=k-$ $M+1$. For $n \in[0, N-1]$, the signal $i_{\text {output }}(t)$ has a spike pulse at the interval of $M-1 \leq k \leq M-1+N-1$. When a sensor code is not identical to the interrogating code, for the orthogonality of the code set, the response signal of the interrogation is irregular and of low amplitude. Thus, a sensor with code $a_{m}=$ $b_{m}$ can be distinguished by interrogating signal with code $\left\{b_{m}\right\}$. Therefore, the collision problem in the SAW multisensor system can be solved by using a correlator on each sensor chip and a group of sensor codes that exhibit high autocorrelation and low cross-correlation properties; this requirement is less stringent than that used in a conventional CDM-based system.

\section{Simulation Experiment}

Taking a SAW multisensor system with six sensors, for example, the sensor code length $Z$ is assumed to be 16 , and the code of each sensor is selected randomly and is shown in Table 1 . The symbol length of the sensor code is 49 periodicity lengths of the IDT. Then the numbers of electrode pairs of the left and the right clusters of IDT on every sensor are set to 10 and 784, respectively.

To discriminate sensor 1 , the interrogating signal shown in Figure 7 is defined as sensor 1 code modulated by the carrier signal. The spaces between the symbols are reserved, to avoid intersymbol interference after IDT transformation. All the sensors receive the interrogating signal, which is transformed into SAW signals by the IDTs, and the output of each IDT is shown in Figure 8. The trapezoidal amplitude of the emerging symbol wave is due to the time delays induced by the IDT length. 


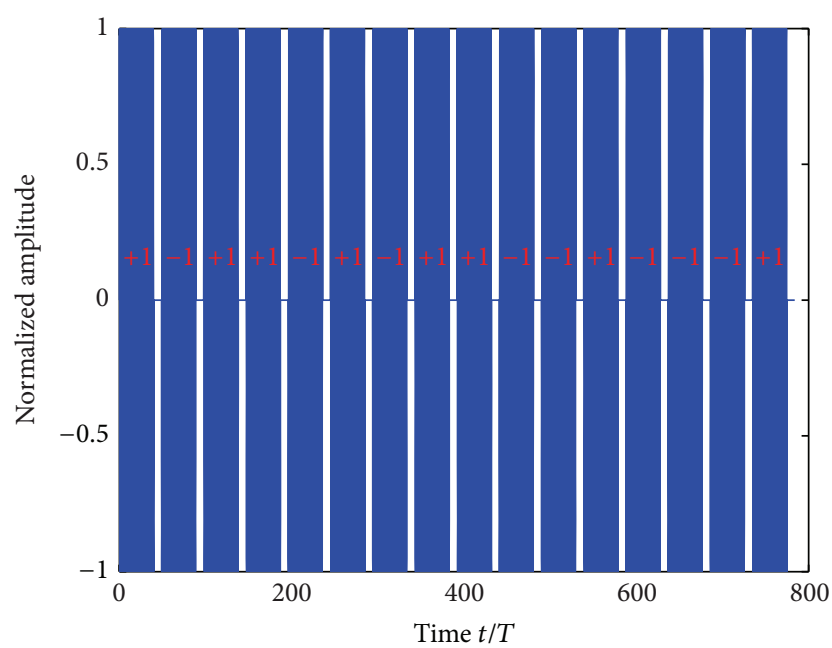

Figure 7: Interrogating signal sent from the reader.

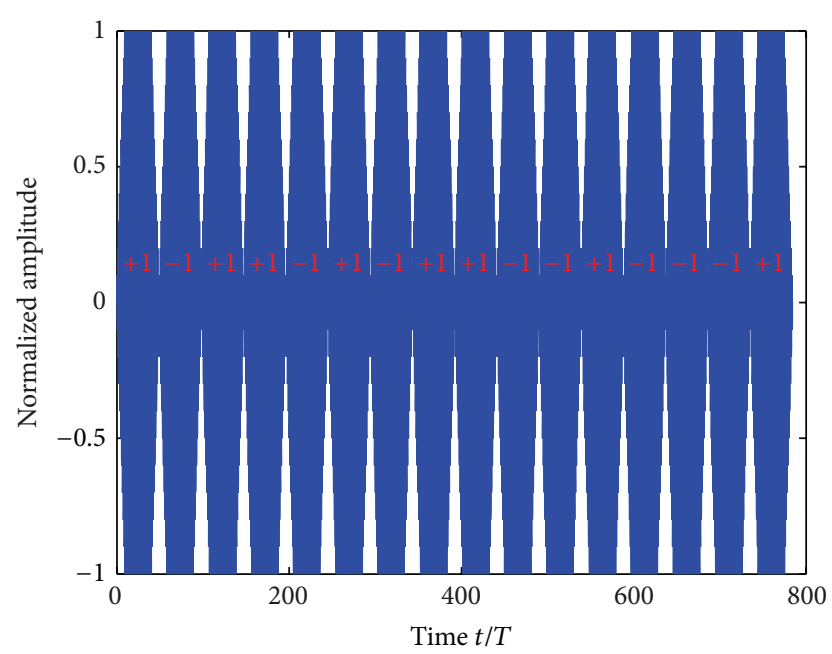

FIGURE 8: Interrogating signal transduced by the IDT.

When the interrogating signal is transformed into SAWs by the IDTs and propagates to the correlators of the sensors, cross-correlations are completed on every sensor chip. All the response signals from every sensor are superposed asynchronously at the reader, owing to the different distances between the sensors and the reader. Based on the proposed signal analysis, the response signal of each sensor was simulated and the delay of the response signal determined randomly. The simulation result is shown in Figure 9. The response signals superposed asynchronously at the reader show a clear spike pulse, which is generated by the autocorrelation of sensor 1 code. Although the response signals from different sensors are asynchronous, sensor 1 can be distinguished by the reader from the response signals.

Similarly, if sensor 2 is required to be discriminated, the interrogating signal used is the sensor 2 code modulated by the carrier signal. The response signals of the sensors arriving at the reader are shown in Figure 10, and a spike pulse verifies the existence of sensor 2. By the same approach, sensor 3

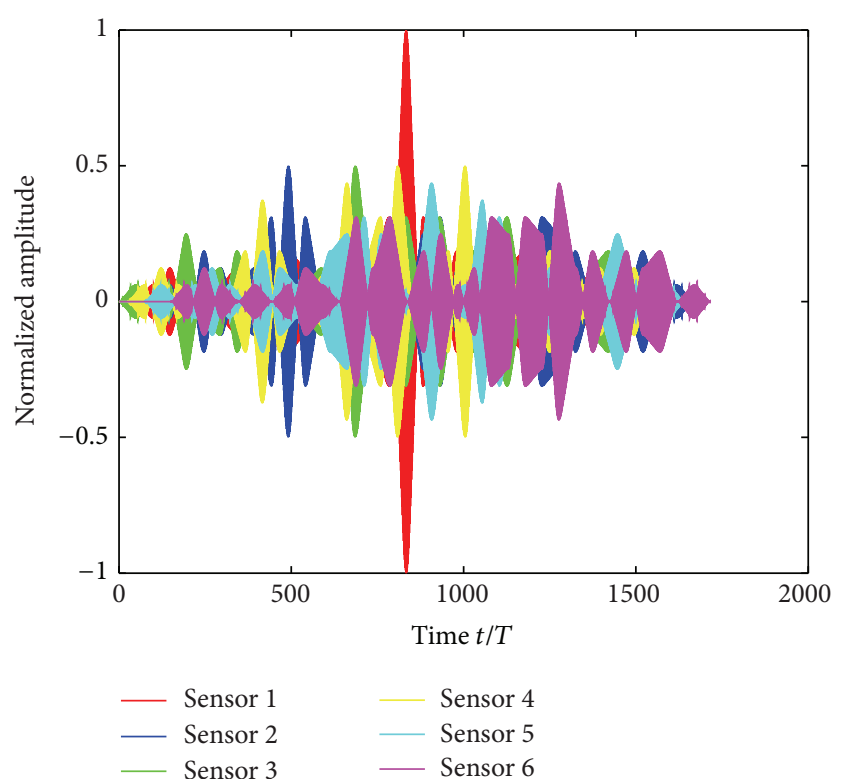

FIGURE 9: Response signals generated by the interrogation with sensor 1 code.

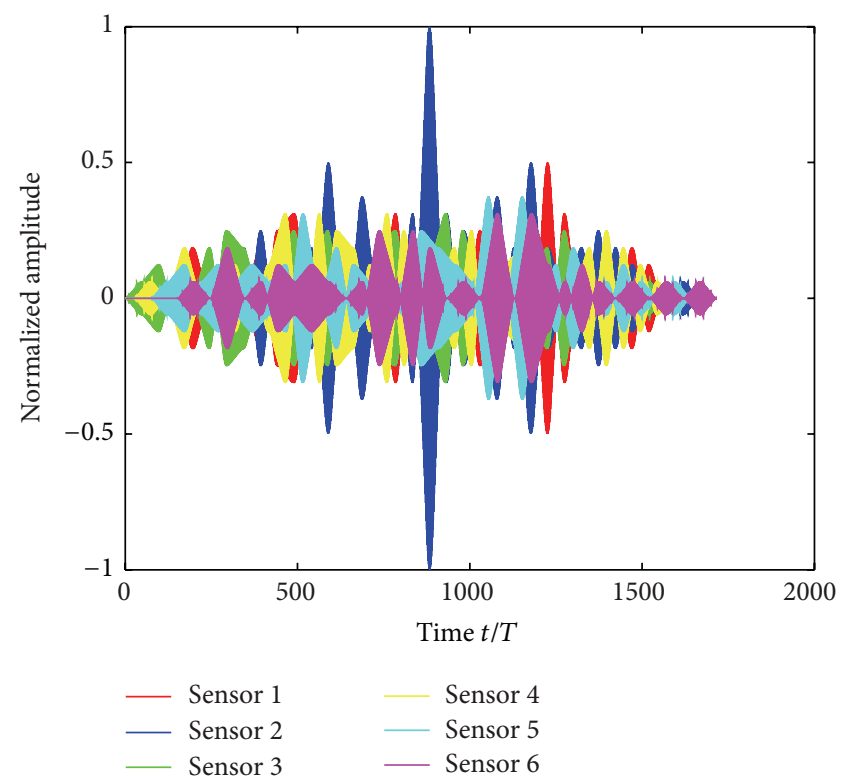

FIGURE 10: Response signals generated by the interrogation with sensor 2 code.

is discriminated by the response signals shown in Figure 11. Therefore, using the on-chip correlator system, the problem of intersensor interference can be solved in a passive wireless SAW multisensor system.

\section{Conclusions}

In conventional CDM-based passive wireless SAW multisensor systems, the response signals from the sensors are distinguished by a correlation operation at the reader. However, for the asynchronous superposition of response signals from 


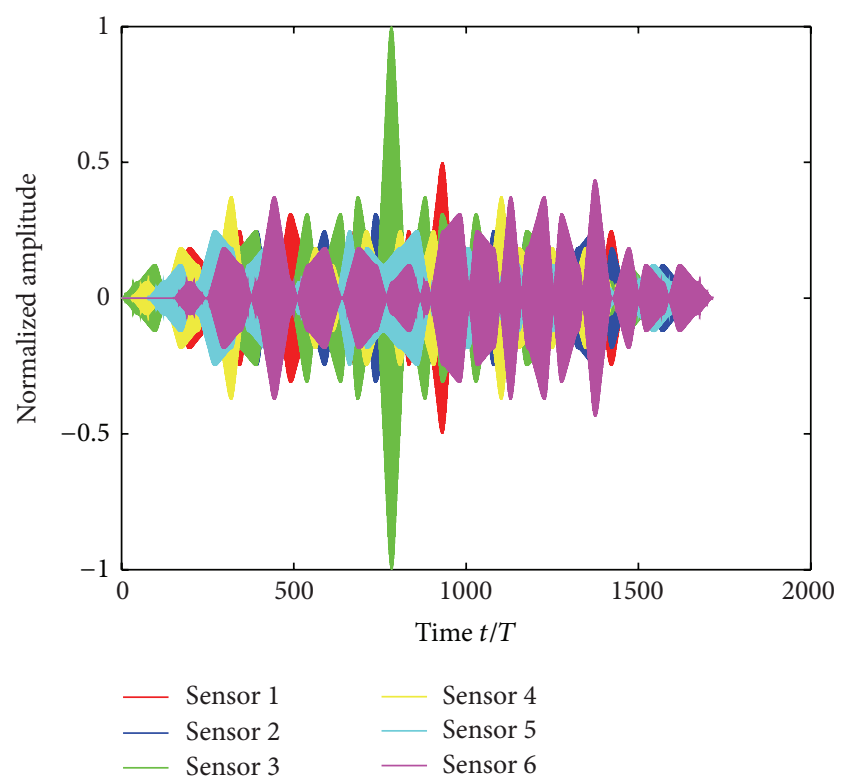

FIGURE 11: Response signals generated by the interrogation with sensor 3 code.

different sensors, it is difficult to achieve this correlation. To solve this problem, an on-chip correlator scheme is demonstrated. In contrast with conventional CDM-based systems, in this novel scheme, correlation takes place at the SAW sensor chip instead of the reader. Then, the response signals arriving at the reader are the results of cross-correlation on the chips. The reader can easily distinguish the sensor that is matched with the interrogating signal. Based on the novel scheme, the operating principle, signal analysis, and simulation are demonstrated. Three examples presented show the response signals from all the sensors for different interrogating signals. There is a clear spike pulse in the response signals, which is generated by the correlation of the sensor code that matches the interrogating signal. The simulations results verify the feasibility of the on-chip correlator concept, which can help to increase the number of simultaneous operational sensors. Future developments will focus on the fabrication process of the SAW correlator chip, performance testing, and potential applications.

\section{Competing Interests}

The authors declare that there is no conflict of interests regarding the publication of this paper.

\section{Acknowledgments}

The authors wish to acknowledge support from the National Natural Science Foundation of China (Grant no. 51505499) and the Jiangsu Province Natural Science Foundation of China (Grant no. BK20150712).

\section{References}

[1] J. H. Hines, "Review of recent passive wireless SAW sensor and sensor-tag activity," in Proceedings of the 4th IEEE Annual
Caneus Fly by Wireless Workshop (FBW '11), pp. 1-2, Montreal, Canada, June 2011.

[2] J. Kim, R. Luis, M. S. Smith, J. A. Figueroa, D. C. Malocha, and B. H. Nam, "Concrete temperature monitoring using passive wireless surface acoustic wave sensor system," Sensors and Actuators A: Physical, vol. 224, pp. 131-139, 2015.

[3] G. A. Borrero, J. P. Bravo, S. F. Mora, S. Velásquez, and F. E. Segura-Quijano, "Design and fabrication of SAW pressure, temperature and impedance sensors using novel multiphysics simulation models," Sensors and Actuators A: Physical, vol. 203, pp. 204-214, 2013.

[4] C. Hartmann, P. Hartmann, P. Brown, J. Bellamy, L. Claiborne, and W. Bonner, "Anti-collision methods for global SAW RFID tag systems," in Proceedings of IEEE International Ultrasonics, Ferroelectrics, and Frequency Control Joint 50th Anniversary Conference, pp. 805-808, August 2004.

[5] V. P. Plessky and L. M. Reindl, "Review on SAW RFID tags," IEEE Transactions on Ultrasonics, Ferroelectrics, and Frequency Control, vol. 57, no. 3, pp. 654-668, 2010.

[6] S. Härmä, W. G. Arthur, C. S. Hartmann, R. G. Maev, and V. P. Plessky, "Inline SAW RFID tag using time position and phase encoding," IEEE Transactions on Ultrasonics, Ferroelectrics, and Frequency Control, vol. 55, no. 8, pp. 1840-1846, 2008.

[7] D. C. Malocha, M. Gallagher, B. Fisher, J. Humphries, D. Gallagher, and N. Kozlovski, "A passive wireless multi-sensor SAW technology device and system perspectives," Sensors, vol. 13, no. 5, pp. 5897-5922, 2013.

[8] J. Xing, W. Liu, and L. Xie, "A novel identification method of multiple OFC SAW sensor tags," in Proceedings of the 10th IEEE International Conference on Nano/Micro Engineered and Molecular Systems (NEMS '15), pp. 187-192, IEEE, Xian, China, April 2015.

[9] A. T. Hines, D. Y. G. Tucker, J. H. Hines, J. Castro, and A. Abedi, "Techniques for optimal DSSS code selection for SAW multisensor systems," in Proceedings of the 66th IEEE International Frequency Control Symposium (IFCS '12), pp. 173-176, May 2012.

[10] K. Hashimoto and M. Yamaguchi, "Derivation of coupling-ofmodes parameters for SAW device analysis by means of boundary element method," in Proceedings of the IEEE Ultrasonics Symposium, pp. 21-26, Orlando, Fla, USA, December 1991. 


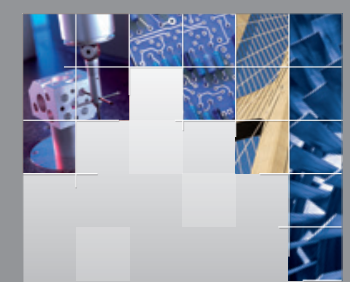

\section{Enfincering}
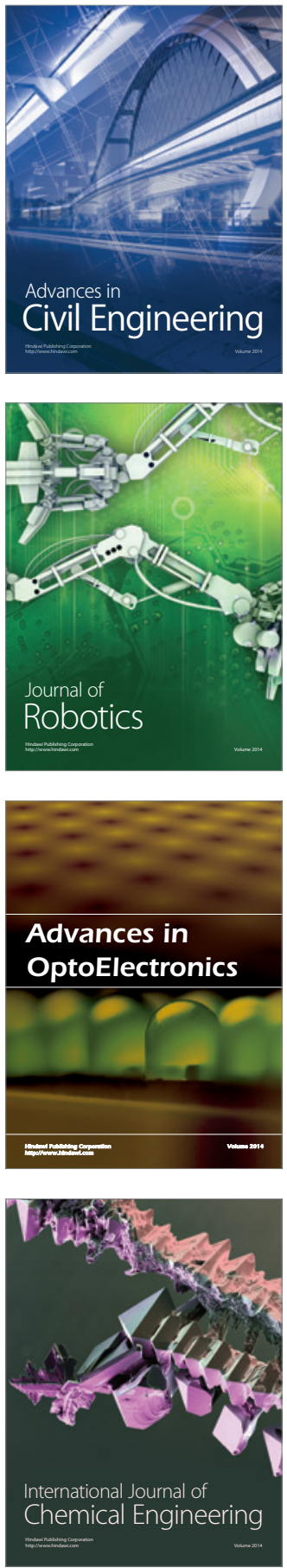

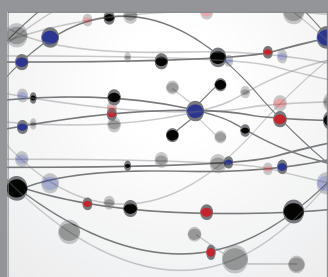

The Scientific World Journal

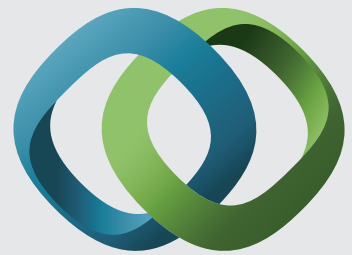

\section{Hindawi}

Submit your manuscripts at

http://www.hindawi.com
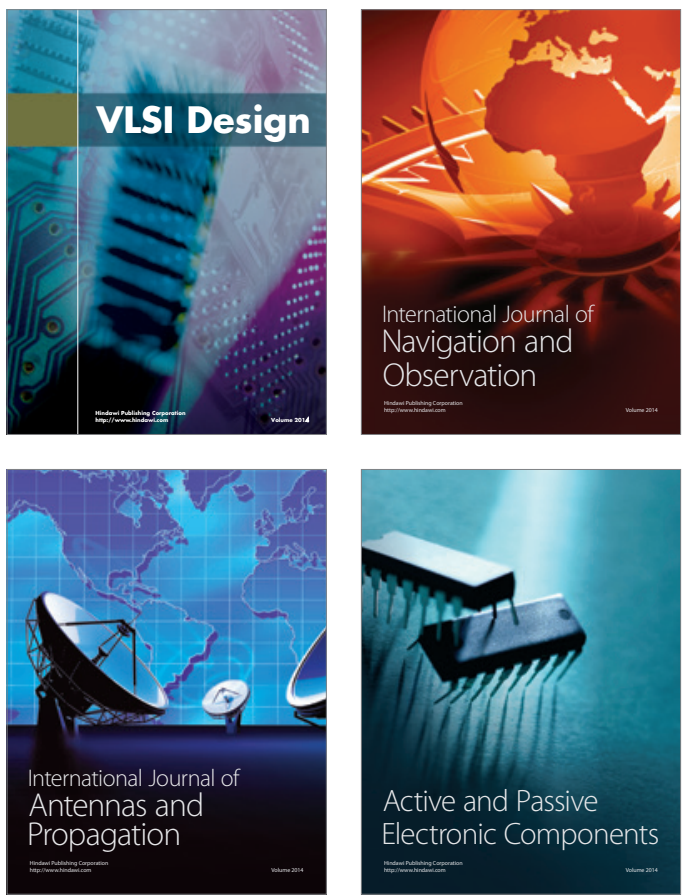
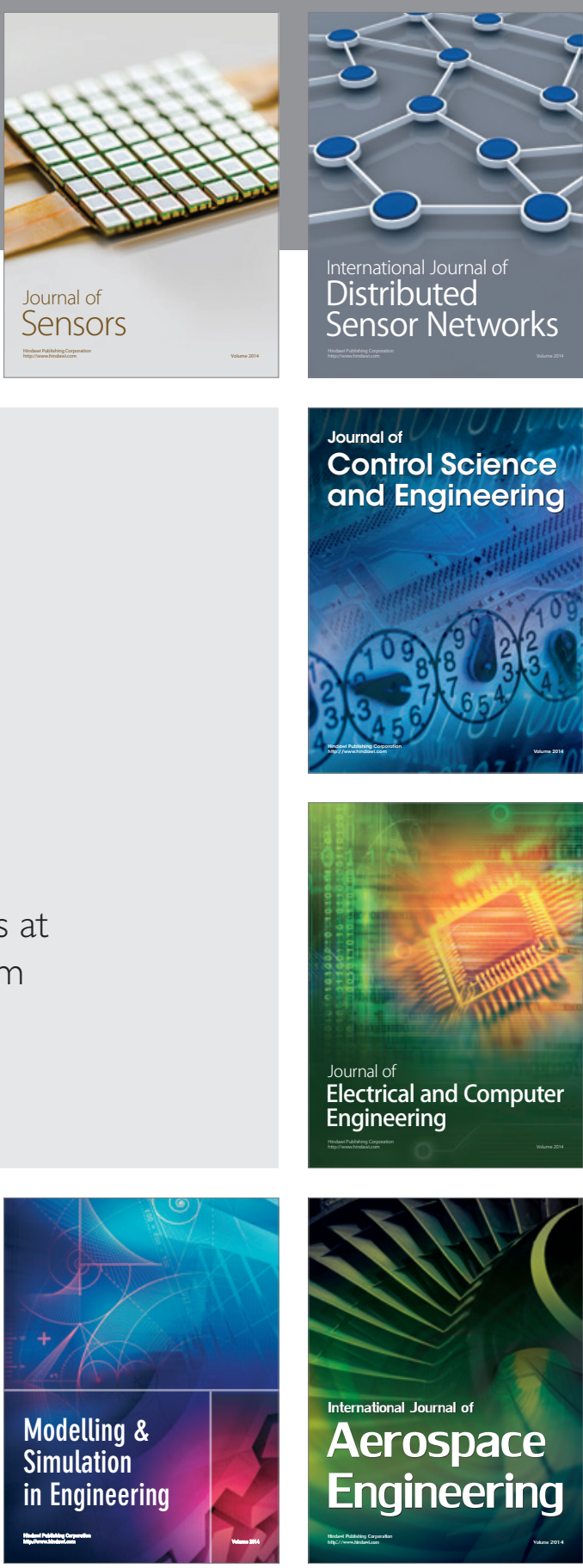

International Journal of

Distributed

Sensor Networks

Journal of

Control Science

and Engineering
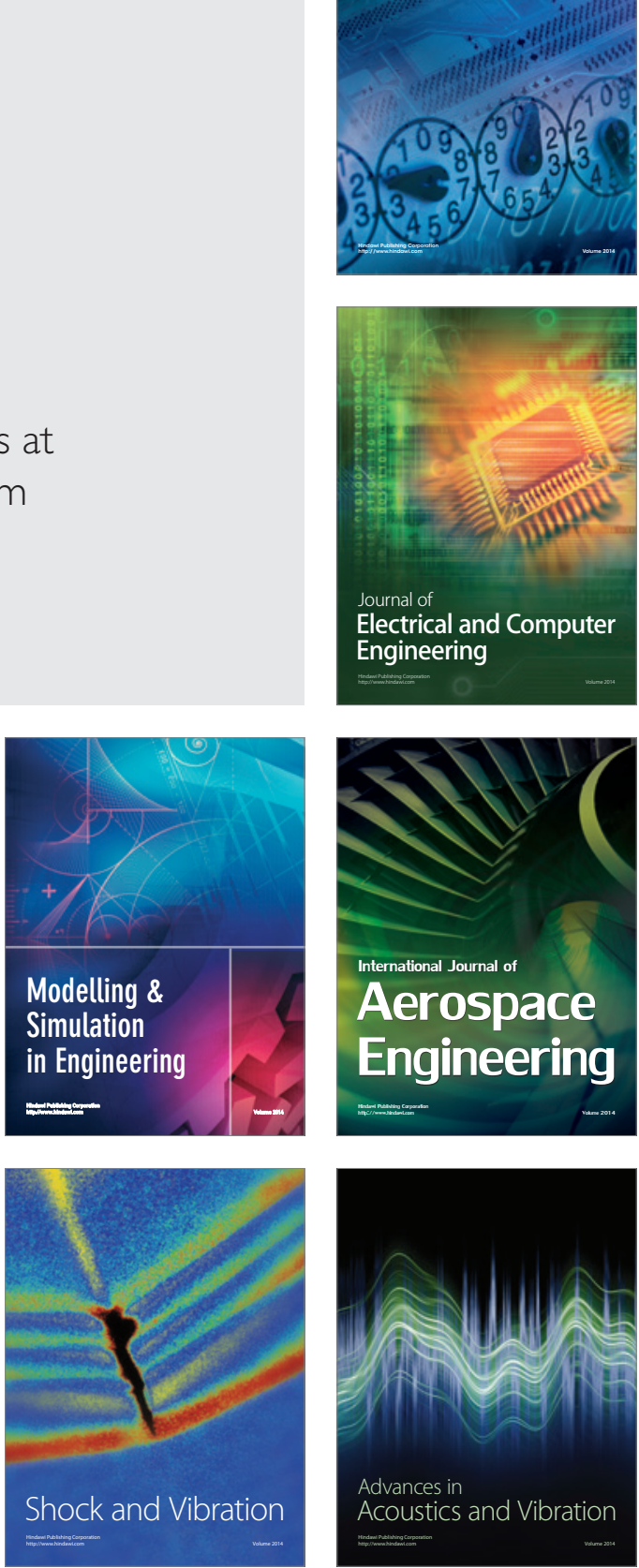